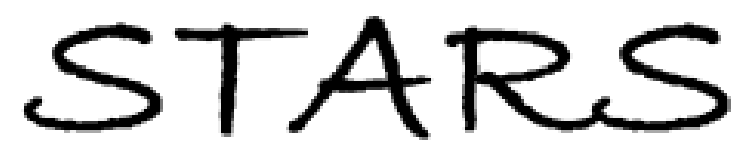

University of Central Florida

STARS

$1-1-1997$

\title{
Spatial soliton robustness against spatially anisotropic phase perturbations
}

Jin U. Kang

C. J. Hamilton

J. S. Aitchison

G. I. Stegeman

University of Central Florida

Find similar works at: https://stars.library.ucf.edu/facultybib1990

University of Central Florida Libraries http://library.ucf.edu

This Article is brought to you for free and open access by the Faculty Bibliography at STARS. It has been accepted for inclusion in Faculty Bibliography 1990s by an authorized administrator of STARS. For more information, please contact STARS@ucf.edu.

\section{Recommended Citation}

Kang, Jin U.; Hamilton, C. J.; Aitchison, J. S.; and Stegeman, G. I., "Spatial soliton robustness against spatially anisotropic phase perturbations" (1997). Faculty Bibliography 1990s. 1963.

https://stars.library.ucf.edu/facultybib1990/1963

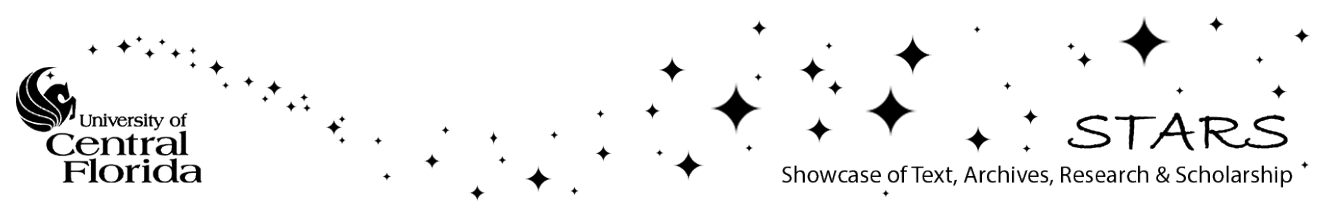




\section{Spatial soliton robustness against spatially anisotropic phase perturbations}

Cite as: Appl. Phys. Lett. 70, 1363 (1997); https://doi.org/10.1063/1.118580

Submitted: 18 November 1996 . Accepted: 13 January 1997 . Published Online: 04 June 1998

Jin U. Kang, C. J. Hamilton, J. S. Aitchison, and G. I. Stegeman

\section{ARTICLES YOU MAY BE INTERESTED IN}

Dark soliton solution of Sasa-Satsuma equation

AIP Conference Proceedings 1212, 114 (2010); https://doi.org/10.1063/1.3367022

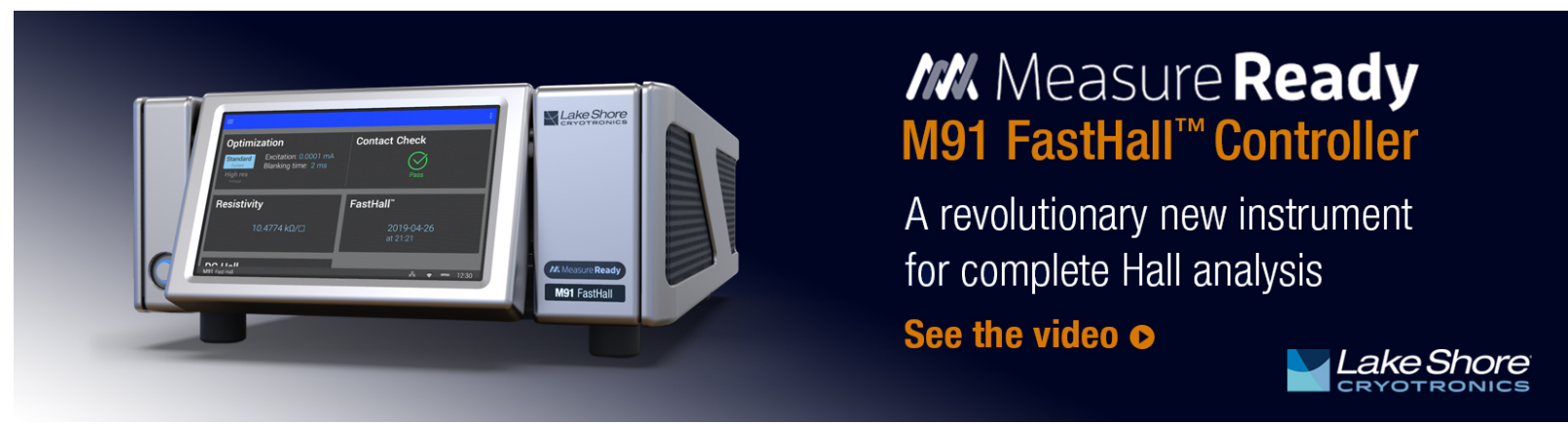




\title{
Spatial soliton robustness against spatially anisotropic phase perturbations
}

Jin U. Kang ${ }^{\mathrm{a})}$

Code 5670, Naval Research Laboratory, Washington, D. C. 20375

C. J. Hamilton and J. S. Aitchison

Department of Electronic and Electrical Engineering, University of Glasgow, Scotland, United Kingdom

G. I. Stegeman

Center for Research and Education in Optics and Lasers (CREOL), University of Central Florida, Orlando, Florida

(Received 18 November 1996; accepted for publication 13 January 1997)

\begin{abstract}
We demonstrate experimentally that spatial solitons in AlGaAs waveguides are resilient against spatially anisotropic perturbations in their phase caused by introducing a wedge in the soliton propagation path. In agreement with numerical simulations, the solitons maintained their initial beam shape and width, independent of the fraction of the soliton beam intercepted by the wedge.
\end{abstract}

(C) 1997 American Institute of Physics. [S0003-6951(97)04211-3]

One dimensional (1D) spatial solitons, stable solutions of the nonlinear Schrodinger equation (NLS), have now been observed in a multimode liquid waveguide made from $\mathrm{CS}_{2}$, a single-mode planar glass waveguide, and an AlGaAs planar waveguide operating at half the band gap. ${ }^{1-3}$ One of the attractive features of solitons in general is their "robustness" which has been discussed for temporal solitons in optical fibers by Menyuk. ${ }^{4}$ That is, a true soliton should retain its solitonic properties when its amplitude or phase is perturbed during propagation. This concept has been demonstrated in fibers for perturbations such as loss, amplification, and frequency filtering. ${ }^{4-6}$ One would expect robustness for spatial solitons against similar perturbations. A key question for soliton-based optical interconnects is how a spatially anisotropic phase chirp across the wavefront of soliton beam would affect its propagation. This would occur, for example, when different parts of a soliton propagate through an anisotropic index perturbation, for example the corner of a prism. Such an electro-optically induced prism can be used to electrically control the deflection of spatial solitons, potentially for application to dynamically reconfigurable interconnects. ${ }^{7}$ In this letter, we report an experimental study of the effect of such perturbations on soliton propagation in an AlGaAs planar waveguide. Specifically, we compare the following cases: only a fraction of the soliton beam experiences a phase change, the whole soliton beam experiences a uniform phase change, and a nonsolitonic beam experiences a partial phase change during the propagation.

The waveguide structure consisted of a high-index, guiding region of $1-\mu \mathrm{m}$-thick $\mathrm{Al}_{0.18} \mathrm{Ga}_{0.82} \mathrm{As}$. The upper and lower cladding regions were made of $1.5-\mu \mathrm{m}$-thick $\mathrm{Al}_{0.30} \mathrm{Ga}_{0.70} \mathrm{As}$ and $4-\mu \mathrm{m}$-thick $\mathrm{Al}_{0.40} \mathrm{GA}_{0.60} \mathrm{As}$, respectively. To test the soliton robustness as discussed above, a triangular wedge was fabricated by standard optical lithography and reactive ion etching of the upper cladding layer down to approximately $0.4 \mu \mathrm{m}$ outside the wedge, see Fig. 1 . The short dimensions of the wedge were 50 by $50 \mu \mathrm{m}$, the apex angle was $45^{\circ}$, and inside the wedge there was an increase in the effective refractive index of approximately 0.022 . This is sufficiently small to produce negligible guided wave reflec-

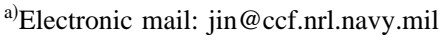

tions at the interfaces, but large enough to give a maximum phase chirp of approximately $1.4 \pi$ across the soliton wavefront. Different amounts of nonuniform phase chirp are introduced by varying the overlap between the solitons and the linear wedge. The total propagation length of the sample was approximately $16.5 \mathrm{~mm}$, and the wedge was formed $4.5 \mathrm{~mm}$ from the input facet to insure that the input soliton would shed its nonsoliton components before it is perturbed by the wedge. Because of the relatively thin upper cladding layer and its etching-induced roughness, the linear loss $\left(0.23 \mathrm{~cm}^{-1}\right)$ of the waveguide was approximately $30 \%$ higher than prior to the etching process. ${ }^{8}$

The experiment was performed with an additively pulse mode-locked $\mathrm{NaCl}: \mathrm{OH}$ color-center laser operating at a wavelength of $1550 \mathrm{~nm}$ with $700 \mathrm{fs}$ pulses at $76 \mathrm{Mhz}$. The laser output was elliptically shaped by a cylindrical telescope, end fire coupled into the waveguide by a $20 \times$ microscope objective, and collected by a $10 \times$ microscope objective at the output. Approximately $16 \pm 5 \mu \mathrm{m}\left(1 / e^{2}\right.$ radius $)$ Gaussian beams were always launched normal to the input facet. The amount of overlap between the soliton beam and the wedge was varied by laterally translating the sample relative to the input beam, see Fig. 1. At the output, the infrared camera was used to record the spatial profiles and the positions of the beams.

At low input powers, the input beam was diffracted by approximately ten times its input width to $160 \pm 30 \mu \mathrm{m}$ after propagation through an unwedged region of the sample, shown in case IV of Fig. 2. When the beam with an input power of $\sim 700 \mathrm{~W}$ was launched, a soliton with the same spatial width as the input beam emerged at the output. This power level is higher than the calculated $\mathrm{cw}$ soliton power $P_{s}$ of $\sim 350 \mathrm{~W}$. However, this is consistent with previous soliton experiments in which power levels of $\sim 1.5 P_{s}$ were required when pulses are used and the increased linear losses in the waveguide. ${ }^{8}$

The three experimental beam geometries are shown in Fig. 1(a): case I when the soliton is not affected by the wedge; case II when half of the soliton is affected by the wedge, and case III when it is fully deflected by the wedge. Corresponding results are shown numerically and experimentally in Figs. 1(b) and 2. When a low power (nonsoliton) 

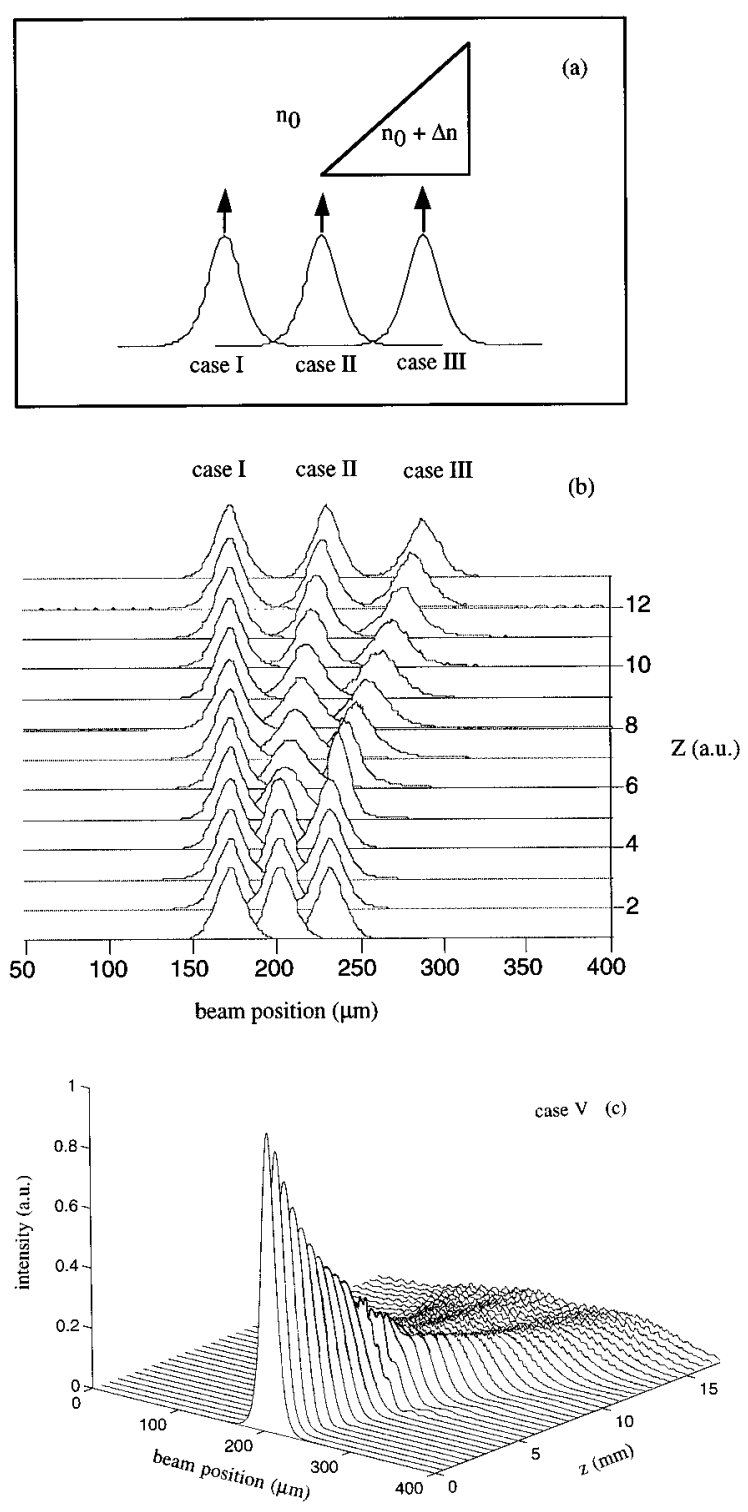

FIG. 1. (a) Schematic of the launching conditions relative to the wedge. (b) Numerical simulations showing the propagation of solitons for the launching conditions shown in (a). (c) Numerical simulations showing the propagation of the nonsoliton beam for the case II.

beam was incident onto the wedge so that half of the beam experienced the chirp due to the wedge and the other half did not, the beam suffered breakup. This is shown numerically and experimentally in Fig. 1(c) and case V of Fig. 2, respectively, by the two main lobes produced on either side of the wedge along with the fringe pattern due to the edge diffraction. The part affected by the wedge was steered according to Snell's Law, the other half propagated in the original direction unaffected by the wedge, and some scattering occurred at the sharp edge. However, for a soliton, the spatially anisotropic phase chirp redistributed itself across the soliton profile so that the soliton maintained its intensity distribution and the whole soliton propagated in a single direction which is intermediate between the original direction and the steered direction for the plane wave case shown in case II of Figs. 1(b) and 2. The shape and the width of the perturbed soliton is within the experimental error identical to the unperturbed case shown in case I. However, notice from Figs. 1(b) and 2 that the output beam in case III is $\approx 20 \%$ wider than the others. This occurs because this soliton experiences a larger perturbation by traveling parallel to, and partially overlapping with the abrupt end of the wedge.

The numerical simulations shown in Fig. 1(b) were in good agreement with experiment, even showing the increase in beam width for case III. They were performed by solving the nonlinear Schrodinger equation (NLS) that describes the paraxial propagation of an optical field in a Kerr medium using a standard fast-Fourier-transform beam propagation method; the nonlinear refractive-index coefficient $n_{2}=1.5 \times 10^{-13} \mathrm{~cm}^{2} / \mathrm{W}$ was used. ${ }^{9}$

A useful prediction of the simulations is that the soliton beam deflection angle is proportional to the fraction of total beam power overlapped with the wedge. This result is expected since larger average phase chirps (increases with increasing beam overlap) lead to larger steering of the beam. Further experiments were performed at spatial overlaps with the wedge of approximately $30 \%$ and $70 \%$, and as discussed previously for the $50 \%$ overlap case, the soliton beam maintained its initial beam shape at the output. The results in Fig. 3 show the net lateral shift as a function of spatial overlap. The spatial overlap is defined as the fraction of the beam's diameter overlapped with the wedge. The measured amount of deflection increases with increasing overlap and is in good agreement with the numerical prediction shown by the dotted line.

In conclusion, we have investigated the propagation of spatial solitons through a linear wedge which introduced a spatially anisotropic linear phase chirp across the soliton beam in an AlGaAs planar waveguide. The results show that solitons are resilient against such perturbations, maintaining their shape and deflecting through an angle determined by the space-averaged phase chirp introduced across the wavefront. This indicates that electro-optically induced index changes in prism electrode geometries can be used to steer spatial soliton beams without beam distortion or breakup.

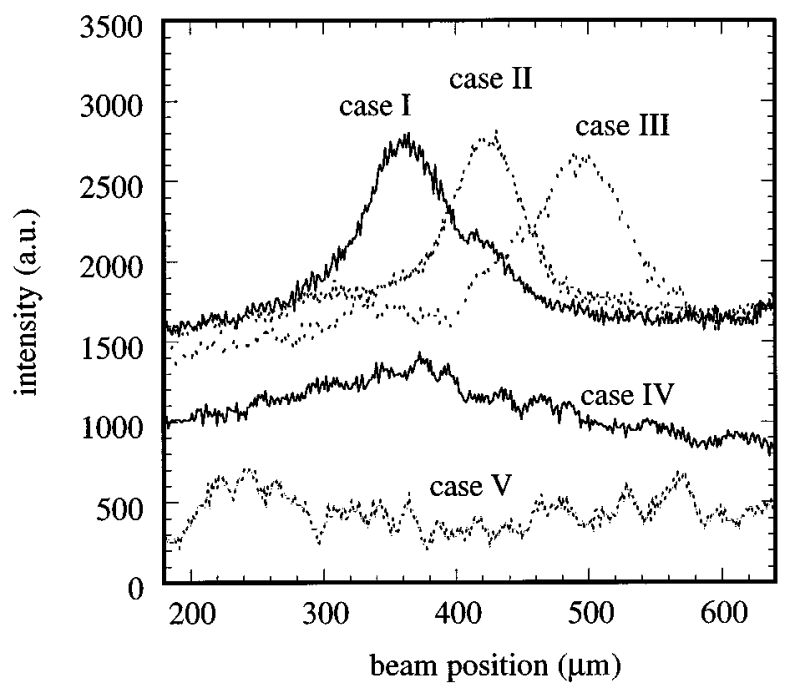

FIG. 2. Experimental results showing the output beam profiles for the different launching cases shown in Fig. 1(a). Case IV and V is for the same launching condition as case I and II, respectively, but for a weak nonsoliton beam. 


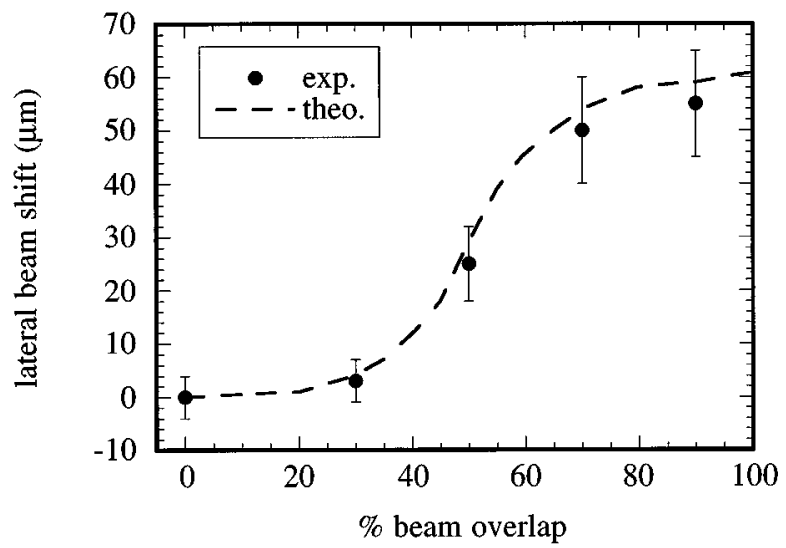

FIG. 3. Lateral shift of the beam relative to the input beam position as a function of spatial overlap between the soliton beam and the linear wedge. Solid dots and the dotted line represent the experimental and theoretical results, respectively.

Since spatial solitons can guide other beams, i.e., signals, they can in principle be used for electrically controlled, dynamically reconfigurable interconnects. However, our numerical studies have indicated that phase ramps greater than
$3 \pi$ can lead to solitonic beam breakup and this aspect needs to be addressed in future experiments.

The research at CREOL was supported by BMDO, NSF, and AFOSR. J. Kang would like to thank C. Menyuk for helpful discussions and the Office of Naval Research for support. J. S. Aitchison would like to thank the Royal Society of Edinburgh/Scottish Office Education Department.

${ }^{1}$ A. Barthelemy, S. Maneuf, and C. Froehly, Opt. Commun. 55, 193 (1985).

2 J. S. Aitchison, A. M. Weiner, Y. Silberberg, M. K. Oliver, J. L. Jackel, D.

E. Leaird, E. M. Vogel, and P. W. E. Smith, Opt. Lett. 15, 471 (1990).

${ }^{3}$ J. S. Aitchison, K. Al-Hemyari, C. N. Ironside, R. S. Grant, and W. Sibbett, Electron. Lett. 28, 1879 (1992).

${ }^{4}$ C. R. Menyuk, J. Opt. Soc. Am. B 10, 1585 (1993).

${ }^{5}$ A. Hasegawa and Y. Kodama, Opt. Lett. 15, 1443 (1990).

${ }^{6}$ A. Mecozzi, J. D. Moores, H. A. Haus, and Y. Lai, Opt. Lett. 16, 1841 (1991).

${ }^{7}$ P. V. Mamyshev, A. Villeneuve, G. I. Stegeman, and J. S. Aitchison, Electron. Lett. 30, 726 (1994).

${ }^{8}$ J. U. Kang, G. I. Stegeman, and J. S. Aitchison, Opt. Lett. 20, 2069 (1995).

${ }^{9}$ A. Villeneuve, C. C. Yang, G. I. Stegeman, C.-H. Lin, and H.-H. Lin, Appl. Phys. Lett. 62, 2465 (1993). 